\title{
Z-GRADUATIONS DE TYPE PARABOLIQUE ET POLYNOMES HARMONIQUES
}

\author{
ABDEL LATIF MORTAJINE
}

\section{A la mémoire de mon père}

\begin{abstract}
We give pluriharmonic and harmonic representations of prehomogeneous vector spaces of regular classical parabolic type $(L=\Gamma \times M, V)$ througt the commutative quotients $V / / M=\operatorname{spec}\left(C[V]^{M}\right)$ of them.
\end{abstract}

\section{$\S 0$. Introduction}

0.1. Soit $(L, \rho, V)$ un triplet composé d'un groupe linéaire algébrique connexe complexe $L$ et d'une représentation rationnelle $\rho$ de $L$ dans un espace vectoriel $V$ de dimension finie $n$ sur $\mathbf{C}$.

$\mathrm{Si}$, dans une base de $V, P(x)=\sum_{i=1}^{n} a_{i_{1}, \ldots, i_{n}} x_{1}^{i_{1}} \cdots x_{n}^{i_{n}}$, on note:

$$
P(\partial)=\sum_{i=1}^{n} \overline{a_{i_{1}, \ldots, i_{n}}}\left(\frac{\partial}{\partial x_{1}}\right)^{i_{1}} \cdots\left(\frac{\partial}{\partial x_{n}}\right)^{i_{n}}
$$

Soit $H$ un sous-groupe de $L$, on note $\mathbf{C}^{H}[V]$ l'algèbre de polynômes $\rho(H)$ invariants et $\mathcal{I}(V)$ l'idéal des polynômes de $\mathbf{C}^{H}[V]$ qui s'annulent en 0 .

Un polynôme $Q \in \mathbf{C}[V]$ est dit harmonique si $P(\partial) Q=0$, pour $P \in$ $\mathcal{I}(V)$.

Dans ce travail, on se place dans le cas où le triplet $(L, \rho, V)$ est un espace préhomogène classique (dont la définition est rappelée ci-dessous), on détermine un sous-groupe $H$ de $L$ et:

i) on montre que tout polynôme sur $V$ s'écrit sous la forme $\sum P Q$ où $P$ est un polynôme $\rho(H)$-invariant et $Q$ un polynôme harmonique.

ii) on décrit les composantes isotypiques de l'action de $L$ sur l'algèbre des polynômes $\mathbf{C}[V]$ sur $V$ définie par: $g \cdot P(x)=P\left(\sigma\left(g^{-1} \cdot x\right)(P(x) \in\right.$ $\mathbf{C}[V]$ et $g \in L)$.

Received February 17, 1997. 
0.2. Dans $[\mathrm{K}-\mathrm{V}]$, Kashiwara et Vergne décrivent les représentations du groupe $G L(n) \times O(k)$ (resp. $G L(p) \times G L(q) \times G L(r))$ dans l'espace de polynômes pluriharmoniques sur l'espaces $M(n, k)(\operatorname{resp.} M(p, q) \oplus M(q, r))$. Ils construisent par la suite, après avoir défini les polynômes harmoniques, des opérateurs d'entrelacements entre les représentations harmoniques et les représentations unitaires sur des espaces symétriques hermitiens $G / K$.

Dans [Ru-Sch], Rubenthaler et Schifmann, en partant des espaces préhomogènes de type parabolique commutatifs, décrivent les représentations harmoniques sur l'espace de la représentation de départ.

Notons que les deux théories généralisent directement la définition classique des polynômes harmoniques; à savoir les polynômes nuls sous l'action de l'opérateur de Laplace.

Dans ce travail, toujours dans le cadre des espaces préhomogènes de type parabolique classique, on construit des opérateurs d'entrelacement avec des représentations des $\mathrm{P}$. H. de type parabolique commutatif (qui sont en bijection avec les espaces symétriques hermitiens $G / K$ ) et nous décrivons les représentations pluriharmoniques.

\section{$\S 1$. Notations}

1.1. Soient $p, q$ deux entiers non nuls; $M(p, q)$ désigne l'algèbre des matrices de type $p \times q$ à coefficients complexes. On note $M(p)$ au lieu de $M(p, p)$. Le groupe des matrices inversibles de $M(p)$ est noté $G L(p)$. On note $H(p)$ l'un des sous-groupes de $G L(p)$ suivants:

$$
\begin{aligned}
& S L(p)=\{g \in G L(p) \mid \operatorname{det}(g)=1\} \\
& S O(p)=\left\{g \in G L(p) \mid g \cdot{ }^{t} g=I_{p}\right\} \\
& \text { avec } I_{p} \text { la matrice identité de } M(p) \text { si } p=2 p^{\prime} \text {, } \\
& S p(p)=\left\{g \in G L(p) \mid g \cdot J_{p}{ }^{t} g=J_{p}\right\} \\
& \text { avec } J_{p}=\left(\begin{array}{cc}
0 & I_{p^{\prime}} \\
-I_{p^{\prime}} & 0
\end{array}\right) \\
& H(2)=D_{2}=\left\{\left(\begin{array}{ll}
a & 0 \\
0 & b
\end{array}\right) \mid a, b \in \mathbf{C}^{*}\right\}
\end{aligned}
$$

1.2. La représentation $\rho_{(p, q)}$ de $G L(p) \times G L(q)$ dans $M(p, q)$ est définie par:

$$
\rho_{(p, q)}(g, h) \cdot x=g \cdot x \cdot h^{-1} \quad(g \in G L(p), h \in G L(q) \text { et } x \in M(p, q))
$$


On note $\tau_{(p, q)}$ la restriction de $\rho_{(p, q)}$ à $G L(p) \times H(q)$. La représentation $2 \Lambda_{p-1}\left(\right.$ resp. $\left.\Lambda_{p-2}\right)$ du groupe $G L(p)$ dans l'espaces $M S(p)$ (resp. $M A S(p)$ ) des matrices symétriques (resp. antisymétriques) est définie par:

$$
\begin{aligned}
2 \Lambda_{p-1}(g) x & =g x^{t} g & & (g \in G L(p) \text { et } x \in M S(p)) \\
\text { (resp. } \quad \Lambda_{p-2}(g) x & =g x^{t} g & & (g \in G L(p) \text { et } x \in M A S(p)))
\end{aligned}
$$

1.3. Soit $r \in \mathbf{N}^{*}$, on note $p(r)=\left(p_{1}, \ldots, p_{r}\right) \in\left(\mathbf{N}^{*}\right)^{r}$. On considère le groupe $G L_{p(r)}=\prod_{i=1}^{r} G L\left(p_{i}\right)$ muni de la représentation:

$$
\rho_{p(r)}=\left[\bigoplus_{i=1}^{r-1} \rho_{\left(p_{i}, p_{i+1}\right)}\right] \text { dans l'espace } V_{p(r)}=\left[\bigoplus_{i=1}^{r-1} M\left(p_{i}, p_{i+1}\right)\right] .
$$

\begin{tabular}{|c|c|c|}
\hline \multicolumn{3}{|c|}{ Tableau 0} \\
\hline$L$ & $\rho$ & $\bar{V}$ \\
\hline$G L_{p(r)} \times H(q)$ & $\rho=\rho_{p(r)} \oplus \tau_{\left(p_{r}, q\right)}$ & $\left.V=V_{p(r)} \oplus M\left(p_{r}, q\right)\right)$ \\
\hline$G L_{p(r)}$ & $\rho=\rho_{p(r)} \oplus 2 \Lambda_{p_{r}-1}$ & $\left.V=V_{p(r)} \oplus M S\left(p_{r}\right)\right)$ \\
\hline$G L_{p(r)}$ & $\rho=\rho_{p(r)} \oplus \Lambda_{p_{r}-2}$ & $\left.V=V_{p(r)} \oplus M A S\left(p_{r}\right)\right)$ \\
\hline
\end{tabular}

1.4. Dans toute la suite le triplet $(L, \rho, V)$ désigne l'un des triplets suivants:

Remarque 1.4.1. A l'exception du cas où $H(q)=D_{2}$, la représentation $\rho$ est irréductible si et seulement si $r=1$.

\section{§2. Les espaces préhomogènes}

2.1. Soit $(G, \sigma, F)$ un triplet composé d'un groupe linéaire algébrique connexe complexe $G$ et d'une représentation rationnelle $\sigma$ de $G$ dans un espace vectoriel $F$ de dimension finie sur $\mathbf{C}$. Un tel triplet est dit espace préhomogène (en abrégé P.H.) s'il existe dans $F$ une orbite Zariski-ouverte $\Omega$. Le sous-ensemble algébrique $S=F-\Omega$ est dit lieu singulier de $(G, \sigma, F)$. Par point générique, on entend les éléments de la grosse orbite $\Omega$ et par sous-groupe d'isotropie générique le sous-groupe d'isotropie $G_{x}=\{g \in G \mid$ $\rho(g) \cdot x=x\}$ avec $x \in \Omega$.

2.2. On note $X(G)$ le groupe des caractères rationnels de $G$. Une fraction rationnelle $f$ sur $F$ est dit un invariant relatif de $(G, \sigma, F)$ s'il existe $\chi \in X(G)$ tel que $f(\sigma(g) \cdot x)=\chi(g) \cdot f(x)$ où $x \in F$ et $g \in G$. Et on dit que $\chi$ est associé à $f$ ou que $f$ est de caractère $\chi$. 
2.3. Soient $S_{0}, \ldots, S_{m}$ les composantes irréductibles de codimension 1 de $S$. On a $S_{k}=\left\{x \in F \mid P_{k}(x)=0\right\}$ avec $P_{k}$ un polynôme irréductible. On sait (voir $[\mathrm{S}-\mathrm{K}]$ ) que $P_{0}, \ldots, P_{m}$ sont des invariants relatifs algébriquement indépendants et que tout invariant relatif $f$ de $(G, \sigma, F)$ s'écrit sous la forme suivante:

$$
f=c \cdot \prod_{i=0}^{m} P_{i}^{m_{i}} \text { avec } c \in \mathbf{C} \quad \text { et } \quad m_{i} \in \mathbf{Z}
$$

On dit que $\left\{P_{0}, \ldots, P_{m}\right\}$ est le système fondamental des invariants relatifs de $(G, \sigma, F)$.

2.4. Un P. H. $(G, \sigma, F)$ est dit régulier s'il existe un invariant relatif $f$ et un point générique $x$ tels que $H_{f}(x) \neq 0\left(H_{f}\right.$ désigne l'Hessien de $\left.f\right)$.

2.5. Deux P. H. $(G, \sigma, F)$ et $\left(G^{\prime}, \sigma^{\prime}, F^{\prime}\right)$ sont dits équivalents s'il existe un isomorphisme rationnel $\xi: \sigma(G) \rightarrow \sigma^{\prime}\left(G^{\prime}\right)$ et un isomorphisme d'espaces vectoriels $\phi: F \rightarrow F^{\prime}$ tels que:

$$
\sigma^{\prime}(\xi(g)) \cdot \phi(x)=\phi(\sigma(g) \cdot x) ; \quad \forall x \in V \quad \text { et } \quad \forall g \in G .
$$

On note $(G, \sigma, F) \cong\left(G^{\prime}, \sigma^{\prime}, F^{\prime}\right)$. Il est clair que deux P. H. équivalents ont les mêmes propriétés.

2.6. On appelle préhomogène réductif tout $\mathrm{P}$. H. dont le groupe $G$ est un groupe réductif. Si le groupe $G$ est réductif, le P. H. est régulier si et seulement si les sous-groupes d'isotropies génériques $G_{x}$ (où $x \in \Omega$ ) sont réductifs (voir $[\mathrm{S}-\mathrm{K}]$ ).

2.7. Un P. H. est dit irréductible si la représentation $\rho$ est irréductible. Un P. H. est dit quasi-irréductible (en abrégé $Q$-irréductible) s'il admet au plus un seul invariant relatif fondamental.

\section{§3. Les espaces préhomogènes de type parabolique}

3.1. Soit $\mathfrak{g}$ une algèbre de Lie réductive complexe de rang $l, \mathfrak{h}$ une sous-algèbre de Cartan de $\mathfrak{g}$ et $R$ le système de racine de la paire $(\mathfrak{g}, \mathfrak{h})$. Soit $\psi=\left\{\alpha_{1}, \ldots, \alpha_{n}\right\}$ une base de $R$ et $\theta$ une partie de $\psi$. On pose $\psi-\theta=$ $\left\{\alpha_{i_{1}}, \ldots, \alpha_{i_{r}}\right\}$. Soit $H_{\theta}$ l'élément défini par:

$$
\alpha\left(H_{\theta}\right)=\left\{\begin{array}{lll}
0 & \text { si } & \alpha \in \theta \\
2 & \text { si } & \alpha \in \psi-\theta
\end{array}\right.
$$

Pour tout $p \in \mathbf{Z}$, on pose $d_{p}(\theta)=\left\{X \in \mathfrak{g} /\left[H_{\theta}, X\right]=2 p X\right\}$. On obtient ainsi une $\mathbf{Z}$-graduation, dite de type parabolique. Il est clair qu'il existe un 
$N_{\theta} \in \mathbf{N}$ tel que $\mathfrak{g}=\bigoplus_{p=-N_{\theta}}^{N_{\theta}} d_{p}(\theta)$. On appelle l'entier $N_{\theta}$ la longueur de la graduation.

3.2. Pour tout $\alpha \in R$, on a: $\alpha=a_{1} \alpha_{1}+\cdots+a_{n} \alpha_{n}$ avec $\left(a_{1}, \ldots, a_{n}\right) \in$ $\left(\mathbf{Z}_{+}\right)^{n}$ ou $_{\mathrm{ex}}\left(a_{1}, \ldots, a_{n}\right) \in\left(\mathbf{Z}_{-}\right)^{n}$. On note $|\alpha|_{\theta}=a_{i_{1}}+\cdots+a_{i_{r}}$ qu'on appelle la $\theta$-longueur de la racine $\alpha$. Si $\mathfrak{g}^{\alpha}$ désigne l'espace raditiel associé à la racine $\alpha$ on vérifie, facilement, que pour tout $p \neq 0$, on a: $d_{p}(\theta)=\bigoplus_{\substack{\alpha \in R \\|\alpha|_{\theta}=p}} \mathfrak{g}^{\alpha}$. Si $\widetilde{\alpha}$ désigne la plus grande racine, alors la longueur de la graduation est donnée par la $\theta$-longueur de $\widetilde{\alpha}$ : $|\widetilde{\alpha}|_{\theta}=N_{\theta}$.

3.3. Soit $G$ le groupe adjoint de $\mathfrak{g}$ et $L_{\theta}$ le centralisateur de $H_{\theta}$ dans $G$, c'est un groupe linéaire, algébrique, connexe et réductif dont $l_{\theta}$ est l'algèbre de Lie. Il est bien connu que l'action adjointe de $L_{\theta}$ stabilise les $d_{p}(\theta)$ et ainsi, définit une représentation dans chaque $d_{p}(\theta)$. On note $\left(L_{\theta}, d_{p}(\theta)\right)$ une telle représentation. Par un résultat connu de Vinberg (voir [V]), la représentation ci-dessus admet un nombre fini d'orbites dont l'une est Zariski-ouverte. (i.e. le triplet $\left(L_{\theta}, d_{p}(\theta)\right)$ est un P. H.).

\section{DÉFINITION 3.3.1.}

i) Le P. H. $\left(L_{\theta}, d_{1}(\theta)\right)$ est dit P. H. de type parabolique. Par extension, on appelle $\mathrm{P}$. H. de type parabolique tout $\mathrm{P}$. H. équivalent à un $\mathrm{P}$. H. de la forme $\left(L_{\theta}, d_{1}(\theta)\right)$.

ii) Un P. H. de type parabolique est dit commutatif si $N_{\theta}=1$.

Remarque 3.3.2. Tout P. H. $\left(L_{\theta}, d_{p}(\theta)\right)$ est un P. H. de type parabolique. (voir [Gy], [Mor 2], [Ru]).

3.4. Décomposition du P. H. $\left(L_{\theta}, d_{1}(\theta)\right)$ En identifiant toute partie de $\psi$ à un sous-diagramme du diagramme de Dynkin, nous donnons un sens aux composantes connexes d'une telle partie. On note $\psi_{k}$ la composante connexe de $\theta \cap\left\{\alpha_{i_{k}}\right\}$ contenant $\alpha_{i_{k}}$. Soit $\theta_{k}=\psi_{k}-\left\{\alpha_{i_{k}}\right\}$ et $l_{k}=\sum_{\gamma \in \psi_{k}} \mathbf{C} H_{\gamma}+\sum_{\gamma \in\left\langle\theta_{k}\right\rangle} \mathfrak{g}^{\gamma}$. On définit l'élément $H_{\theta}^{k} \in \sum_{\gamma \in \psi_{k}} \mathbf{C} H_{\gamma}$ par: $\alpha\left(H_{\theta}^{k}\right)=\left\{\begin{array}{l}0 \text { si } \alpha \in \theta_{k} \\ 2 \text { si } \alpha=\alpha_{i_{k}}\end{array}\right.$ On a $d_{1}\left(\theta_{k}\right)=\left\{X \in \mathfrak{g} /\left[H_{\theta}^{k}, X\right]=2 X\right\}$ et $l_{\theta}=\sum_{k=1}^{r+1} l_{k}$ (la somme n'est pas directe).

THÉORÈmE 3.4.1. ([Ru])

i) L'espace vectoriel $d_{1}(\theta)=\bigoplus_{k=1}^{r} d_{1}\left(\theta_{k}\right)$ et chaque $d_{1}\left(\theta_{k}\right)$ est $l_{\theta}$-stable et irréductible sous l'action de $l_{\theta}$ (aussi celle de $l_{k}$ ). 
ii) Les éléments $H_{\theta}^{k}$ forment une base de $\mathfrak{h}_{\theta}$.

Remarque 3.4.2. $\quad$ Le P. H. $\left(L_{\theta}, d_{1}(\theta)\right)$ est irréductible si et seulement si $(\psi-\theta)$ contient une seule racine (i.e. $r=1)$.

\subsection{Les $\mathbf{P}$. H. de type parabolique classique réguliers}

DÉFinition 3.5.1. On dit qu'un P. H. de type parabolique est classique si l'algèbre de Lie $\mathfrak{g}$ est classique (le diagramme de Dynkin est de type $A_{n}, B_{n}, C_{n}$ ou $D_{n}$ ).

Proposition 3.5.2. Le P. H. $\left(L_{\theta}, d_{1}(\theta)\right)$ est de type parabolique classique si et seulement s'il est équivalent à l'un des triplets $(L, \rho, V)$.

Pour la démonstration voir [Mor 1].

Liste des P. H. étudiés et leur type 3.5.3. Dans le tableau 1, on dresse la liste des triplets considérés dans ce travail et on distingue les différents cas selon le type de l'algèbre de lie de départ $\mathfrak{g}$ et la longueur de la graduation $N_{\theta}$ qui dépend des racines dans $\psi-\theta$ et de leur nombre.

Tableau 1

\begin{tabular}{|c|c|c|c|c|}
\hline$L$ & $\rho$ & $\left(p_{r}, q\right)$ & type & $N_{\theta}$ \\
\hline$G L_{p(r)} \times S L(q)$ & $\rho_{p(r)} \oplus \tau_{\left(p_{r}, q\right)}$ & & $A$ & $r$ \\
\hline$G L_{p(r)} \times S O(q)$ & $\rho_{p(r)} \oplus \tau_{\left(p_{r}, q\right)}$ & $p_{1}=1, q$ impair & $B^{1}$ & $2(r-1)+1$ \\
\hline$G L_{p(r)} \times S O(q)$ & $\rho_{p(r)} \oplus \tau_{\left(p_{r}, q\right)}$ & $p_{1}=1, q$ pair & $D^{1}$ & $2(r-1)+1$ \\
\hline$G L_{p(r)} \times S O(q)$ & $\rho_{p(r)} \oplus \tau_{\left(p_{r}, q\right)}$ & $p_{1}>1, q$ impair & $B^{2}$ & $2 r$ \\
\hline$G L_{p(r)} \times S O(q)$ & $\rho_{p(r)} \oplus \tau_{\left(p_{r}, q\right)}$ & $p_{1}>1, q$ pair & $D^{2}$ & $2 r$ \\
\hline$G L_{p(r)} \times S p(q)$ & $\rho_{p(r)} \oplus \tau_{\left(p_{r}, q\right)}$ & $q$ pair & $C^{1}$ & $2 r$ \\
\hline$G L_{p(r)} \times D_{2}$ & $\rho_{p(r)} \oplus \tau_{\left(p_{r}, 2\right)}$ & $p_{1}=1$ & $D^{5}$ & $2(r-1)+1$ \\
\hline$G L_{p(r)} \times D_{2}$ & $\rho_{p(r)} \oplus \tau_{\left(p_{r}, 2\right)}$ & $p_{1}>1$ & $D^{4}$ & $2 r$ \\
\hline$G L_{p(r)}$ & $\rho_{p(r)} \oplus 2 \Lambda_{p_{r}-1}$ & & $C^{2}$ & $2(r-1)+1$ \\
\hline$G L_{p(r)}$ & $\rho_{p(r)} \oplus \Lambda_{p_{r}-2}$ & & $D^{3}$ & $2(r-1)+1$ \\
\hline$G L_{p(r)}$ & $\rho_{p(r)} \oplus \Lambda_{p_{r}-2}$ & $p_{1}=1$ & $D^{6}$ & $2(r-1)$ \\
\hline
\end{tabular}

Remarque 3.5.4. Mis à part les cas $D^{4}, D^{5}$ et $D^{6}$, Il est clair que $N_{\theta}=1 \Rightarrow r=1$ (la réciproque est fausse).

Remarque 3.5.5. La régularité du P. H. $(L, \rho, V)$ est équivalente aux relations entre les $p_{i}$ (pour $1 \leq i \leq r$ ) et $q$.

Nous renvoyons le lecteur à [Mor 1] pour plus de détails. 


\section{§4. L'application $\pi$}

\subsection{Théorème de l'application $\pi$}

ThÉORÈme 4.1.1. 1) Pour tout P. H. de type parabolique classique $(L, \rho, V)$, Il existe un sous-groupe $\Gamma$ de $L$, une représentation $(\tau, U) d u$ groupe $\Gamma$ (donc de $L)$ et des polynômes homogènes non nuls, de degré $N_{\theta}$, $\pi_{1}(x), \ldots, \pi_{k}(x)$ dans $\mathbf{C}[V]($ avec $k=\operatorname{dim} U)$ tels que:

i) $(\Gamma, \tau, U)$ soit un $P$. H. de type parabolique classique commutatif.

ii) L'application $\pi: V \rightarrow U$ définie par $\pi(x)=\left(\pi_{1}(x), \ldots, \pi_{k}(x)\right)$ est $L$ équivariante, i.e.: $\pi(g . x)=\rho(g) \pi(x) ; \forall g \in L, \forall x \in V$.

iii) On a $L=\Gamma \times M$ et l'algèbre $\mathbf{C}^{M}[V]$ des polynômes $M$-invariants est negendré par $\pi_{1}(x), \ldots, \pi_{k}(x)$.

iv) L'application $\pi^{*}: \mathbf{C}[U] \rightarrow \mathbf{C}[V]$ définie par: $\pi^{*}(P(x))=(P o \pi)(x)$ est un homomorphisme d'algèbres.

Démonstration. (voir [Mor 2]) Le tableau 2, rappelle, dans chaque cas le triplet $(\Gamma, \tau, U)$ et l'application $\pi$. Par une sipmle comparaison de $L$ et de $\Gamma$, on déduit la forme du sous-groupe $M$. Si $\left(x_{1}, \ldots, x_{r-1}\right) \in V_{p(r)}$, on pose $X=x_{1} \cdots x_{r-1}$ et on note $\left(x_{1}, \ldots, x_{r-1}, y\right)$ un élément de $V=V_{p(r)} \oplus E$. Si $y \in M\left(p_{r-1}, 2\right)$, on pose $y=\left(y_{1}, y_{2}\right)$ où $y_{i} \in M\left(p_{r-1}, 1\right)$.

Tableau 2

\begin{tabular}{|c|c|c|c|c|}
\hline Type & $\Gamma$ & $\tau$ & $U$ & $\pi$ \\
\hline$A$ & $G L\left(p_{1}\right) \times S L(q)$ & $\tau_{\left(p_{1}, q\right)}$ & $M\left(p_{1}, q\right)$ & $X \cdot y$ \\
\hline$B^{1}$ & $G L\left(p_{1}\right) \times S O(q)$ & $\tau_{\left(p_{1}, q\right)}$ & $M\left(p_{1}, q\right)$ & $X \cdot y$ \\
\hline$B^{2}$ & $G L\left(p_{1}\right)$ & $2 \Lambda_{p_{1}-1}$ & $M S\left(p_{1}\right)$ & $(X \cdot y)^{t}(X y)$ \\
\hline$C^{1}$ & $G L\left(p_{1}\right)$ & $\Lambda_{p_{1}-2}$ & $M A S\left(p_{1}\right)$ & $(X \cdot y) J_{q}{ }^{t}(X y)$ \\
\hline$C^{2}$ & $G L\left(p_{1}\right)$ & $2 \Lambda_{p_{1}-1}$ & $M S\left(p_{1}\right)$ & $X \cdot y \cdot{ }^{t} X$ \\
\hline$D^{1}$ & $G L\left(p_{1}\right) \times S O(q)$ & $\tau_{\left(p_{1}, q\right)}$ & $M\left(p_{1}, q\right)$ & $X \cdot y$ \\
\hline$D^{2}$ & $G L\left(p_{1}\right)$ & $2 \Lambda_{p_{1}-1}$ & $M S\left(p_{1}\right)$ & $(X \cdot y)^{t}(X y)$ \\
\hline$D^{3}$ & $G L\left(p_{1}\right)$ & $\Lambda_{p_{1}-2}$ & $M A S\left(p_{1}\right)$ & $X \cdot y \cdot{ }^{t} X$ \\
\hline$D^{4}$ & $G L\left(p_{1}\right) \times \mathbf{C}^{*}$ & $\tau_{\left(, p_{1}\right)}$ & $M\left(p_{1}\right)$ & $\left(X \cdot y_{1}\right)^{t}\left(x y_{2}\right)$ \\
\hline$D^{5}$ & $G L\left(p_{2}\right) \times\left(\mathbf{C}^{*}\right)^{2}$ & $\tau_{\left(p_{2}, 2\right)}$ & $M\left(p_{2}, 2\right)$ & $\left(X \cdot y_{1}\right)^{t}\left(x_{2} \ldots x_{r-1} y_{2}\right.$ \\
\hline$D^{6}$ & $G L\left(p_{1}\right) \times S L\left(p_{2}\right)$ & $\tau_{\left(p_{1}, p_{2}\right)}$ & $M\left(p_{1}, p_{2}\right)$ & $\left(X \cdot y_{1}\right)^{t}\left(x_{2} \ldots x_{r-1} y_{2}\right.$ \\
\hline
\end{tabular}

Remarque 4.1.2. Si le P. H. $(L, \rho, V)$ est régulier, le P. H. commutatif $(\Gamma, \tau, U)$ est régulier et l'application polynômiale $L$-équivariante $\pi: V \rightarrow U$ est surjective. Dans ce cas $\pi^{*}$ est injective et par conséquent $\mathbf{C}^{M}[V]$ est isomorphe à l'algèbre $\mathbf{C}[U]$. 
Remarque 4.1.3. Si $(L, \rho, V)$ est un P. H. commutatif, alors l'application $\pi$ est l'application identique de $V$.

4.2. La décomposition de $\mathbf{C}[\pi]$ Dans toute la suite le triplet $(L, \rho, V)$ est régulier.

Rappels et Notation 4.2.1.

i) On note $\mathbf{C}[\pi]=\operatorname{Im}\left(\pi^{*}\right)=\mathbf{C}^{M}[V]$. C'est la sous-algèbre engendrée $\operatorname{par} \pi_{1}, \ldots, \pi_{k}$.

ii) Le P. H. $(\Gamma, \tau, U)$ admet, à la multiplication par un scalaire près, un seul invariant relatif fondamental. On note $\Delta_{0}$ cet invariant relatif, $\chi_{0}$ le caractère correspondant et $H=K e r \chi_{0}$.

On pose $d+1=$ le degré de $\left(\Delta_{0}\right)$ et $k=\operatorname{dim}(U)$.

iii) le P. H. $(\Gamma, \tau, U)$ admet $d+2$ orbites.

iv) Soit $\Omega$ l'orbite ouverte de $(\Gamma, \tau, U)$ et soit $I_{0} \in \Omega$.

Le sous-groupe d'isotropie $S=\Gamma_{I_{0}}$ est réductif.

v) Soit $B$ un sous-groupe de Borel de $\Gamma$, le triplet $(B, \tau, U)$ est un P. H. régulier dont le cardinal du système fondamental des invariants relatifs est égal à $d+1$.

On note $\left\{\Delta_{0}, \Delta_{1}, \ldots, \Delta_{d}\right\}$ ce système (où on les a rangé par ordre décroissant de leurs degrés).

vi) la représentation $(\Gamma, \mathbf{C}[U])$ se décompose avec multiplicité un.

Pour $a=\left(a_{0}, \ldots, a_{d}\right) \in \mathbf{N}^{d+1}$, on note $U_{a}=U_{\left(a_{0}, \ldots, a_{d}\right)}$ le sous$\Gamma$-module de $\mathbf{C}[U]$ engendré par $\Delta_{0}^{a_{0}} \Delta_{1}^{a_{1}} \cdots \Delta_{d}^{a_{d}}$. On a: $\mathbf{C}[U]=$ $\bigoplus_{a \in \mathbf{N}^{d+1}} U_{a}$.

vii) Chaque représentation $\left(\Gamma, U_{a}\right)$ admet un unique vecteur $S$-invariant.

viii) Un polynôme $P(X) \in \mathbf{C}[U]$ est dit harmonique si $\Delta_{0}^{*}(\partial) \cdot P(X)=0$.

On note $\mathcal{H}[U]$ l'algébre des polynômes harmoniques et on rappelle que:

$$
\mathcal{H}[U]=\bigoplus_{\left(a_{1}, \ldots, a_{d}\right) \in \mathbf{N}^{d}} U_{\left(0, a_{1}, \ldots, a_{d}\right)}
$$

et ainsi, tout $P \in \mathbf{C}[U]$ s'écrit d'une manière unique sous la forme:

$$
P(X)=\sum_{\alpha} \Delta_{0}^{\alpha}(X) Q(X) \quad \text { avec } \quad Q \in \mathcal{H}[U]
$$

THÉORÈmE 4.2.2. La représentation $(L, \mathbf{C}[\pi])$ se décompose avec multiplicité un et chaque représentation irréductible intervenant dans la décomposition de $\mathbf{C}[\pi]$ est paramétrée par des entiers $\left(a_{0}, \ldots, a_{d}\right) \in \mathbf{N}^{d+1}$ tels que $\left(\Delta_{0} o \pi\right)^{a_{0}} \cdots\left(\Delta_{d} O \pi\right)^{a_{d}}$ soit le vecteur dominant de la représentation. 
On note $V_{a_{0}, \ldots, a_{d}}$ une telle composante et on a $\mathbf{C}[\pi]=\bigoplus_{\left(a_{0}, \ldots, a_{d}\right) \in N^{d+1}}$ $V_{a_{0}, \ldots, a_{d}}$.

Pour la démonstration voir [Mor 2].

\section{$\S 5$. Le polynôme de Bernstein-Sato}

THÉORÈme 5.1. On note $\Delta_{0}^{\theta}=\Delta_{0}$ o $\pi$ :

i) Il existe un polynôme $\mathcal{B}_{\theta}$ de degré $N_{\theta}(d+1)$ tel que:

$$
\Delta_{0}^{\theta}(\partial) \cdot\left[\Delta_{0}^{\theta}\right]^{s}(X)=\mathcal{B}_{\theta}(s) \cdot\left[\Delta_{0}^{\theta}\right]^{s-1}(X) ; \quad s \in \mathbf{C}
$$

ii) Il existe un polynôme $\mathcal{B}$ de degré $(d+1)$ tel que:

$$
\begin{gathered}
\Delta_{0}(\partial) \cdot \Delta_{0}^{s}(X)=\mathcal{B}(s) \cdot \Delta_{0}^{s-1}(X) ; \quad s \in \mathbf{C} \\
\text { avec } \quad \mathcal{B}(s)=\prod_{j=0}^{d}\left(s+j \cdot \frac{k^{\prime}}{2}\right) ; \quad k^{\prime}=\frac{2}{d(d+1)}(k-d-1)
\end{gathered}
$$

Démonstration.

i) C'est un résultat de $[\mathrm{S}-\mathrm{K}]$.

ii) C'est un résultat de [Mu-Ru-Sch].

ThÉORÈme 5.2. Il existe un polynôme $b_{i}$ de degré $N_{\theta}$ tel que:

$$
\pi_{i}(\partial) \cdot \pi_{j}^{s}(X)=\delta_{i j} b_{i}(s) \cdot \pi_{j}^{s-1}(X) ; \quad s \in \mathbf{C} \text { et } \delta_{i j}=\left\{\begin{array}{lll}
1 & \text { si } & i=j \\
0 & \text { si } & i \neq j
\end{array}\right.
$$

Démonstration. i) Dans le cas $A$ : on a $p_{1}=q$, donc $k=q^{2}$ (car $\left.U=M\left(p_{1}, q\right)=M(q)\right)$. Les polyômes $\pi_{i j}(x)$ sont les coefficients de la matrice $\pi(x)(x \in V)$. On pose $x_{1}=\left(u_{1}, \ldots, u_{q}\right) \in M\left(q, p_{2}\right)$ où $u_{i}$ désigne le ième vecteur ligne de la matrice $x_{1}$. On pose $x_{r}=\left(v_{1}, \ldots, v_{q}\right) \in M\left(p_{r-1}, q\right)$ où $v_{i}$ désigne le ième vecteur colonne de la matrice $x_{r}$. Il est clair que $\pi_{i j}(x)=u_{i} \cdot x_{2} \cdots x_{r-1} \cdot v_{j}$ (pour $\left.1 \leq i, j \leq q\right)$. Donc, $\pi_{i j}(\partial)\left[\pi_{k l}(x)\right]^{s}=0$ si et seulement si $(i, j) \neq(k, l)$. Si on considère le P. H.:

$$
\left(L^{\prime}=\mathbf{C}^{*} \times \prod_{l=2}^{r} G L\left(p_{l}\right), \rho_{\left(1, p_{2}\right)} \oplus \bigoplus_{l=2}^{r-1} \rho_{\left(p_{l}, p_{l+1}\right)} \oplus \tau_{\left(p_{r}, 1\right)}, V=M\left(1, p_{2}\right) \oplus\right.
$$
$\bigoplus_{l=2}^{r-1} M\left(p_{l}, p_{l+1}\right) \oplus M\left(p_{r}, 1\right)$ ) (qui est aussi de type $A$ ), on vérifie facilement que le polynôme $\pi_{i j}$ en est un invariant relatif. D'où l'existence du polynôme de Bernstein-Sato associé au polynôme $\pi_{i j}$.

Notons que dans ce cas $b_{(i, j)}(s)=b_{(k, l)}(s)$ pour $1 \leq i, j, k, l \leq q$. 
ii) Dans le cas $B^{1}$ et $D^{1}$ : on a $p_{1}=1$, donc $k=q(\operatorname{car} U=M(1, q))$. Les polyômes $\pi_{i}(x)$ sont les coefficients de la matrice $\pi(x)(x \in V)$. On pose $x_{r}=\left(v_{1}, \ldots, v_{q}\right) \in M\left(p_{r-1}, q\right)$ où $v_{i}$ désigne le ième vecteur colonne de la matrice $x_{r}$. Il est clair que $\pi_{j}(x)=x_{1} \cdot x_{2} \cdots x_{r-1} \cdot v_{j}$ (pour $1 \leq j \leq q$ ). Donc, $\pi_{j}(\partial)\left[\pi_{l}(x)\right]^{s}=0$ si et seulement si $j \neq l$. Si on considère le P. H.:

$\left(L^{\prime}=\mathbf{C}^{*} \times \prod_{l=2}^{r} G L\left(p_{l}\right), \rho_{\left(1, p_{2}\right)} \oplus \bigoplus_{l=2}^{r-1} \rho_{\left(p_{l}, p_{l+1}\right)} \oplus \tau_{\left(p_{r}, 1\right)}, V=M\left(1, p_{2}\right) \oplus\right.$ $\bigoplus_{l=2}^{r-1} M\left(p_{l}, p_{l+1}\right) \oplus M\left(p_{r}, 1\right)$ ) (qui est de type $A$ ), on vérifie facilement que le polynôme $\pi_{j}$ en est un invariant relatif. D'où l'existence du polynôme de Bernstein-Sato associé au polynôme $\pi_{j}$.

Notons que dans ce cas $b_{j}(s)=b_{l}(s)$ pour $1 \leq i, j, k, l \leq q$.

iii) Dans le cas $B^{2}$ et $D^{2}$ : on a $p_{1}>1$, donc $k=\frac{p_{1}\left(p_{1}+1\right)}{2}$ (car $U=$ $\left.M S\left(p_{1}\right)\right)$. Les polyômes $\pi_{(i, j)}(x)$ sont les coefficients de la matrice $\pi(x)(x \in$ $V)$. On pose $x_{1}=\left(u_{1}, \ldots, u_{p_{1}}\right) \in M\left(p_{1}, p_{2}\right)$ où $u_{i}$ désigne le ième vecteur ligne de la matrice $x_{1}$. Il est clair que $\pi_{(i, j)}(x)=u_{i} \cdot\left(x_{2} \cdots x_{r}\right) \cdot{ }^{t}\left(x_{2} \cdots x_{r}\right)^{t} u_{j}$ $\left(1 \leq i \leq j \leq p_{1}\right)$. Donc, $\pi_{(i, j)}(\partial)\left[\pi_{(k, l)}(x)\right]^{s}=0$ si et seulement si $(i, j) \neq$ $(k, l)$.

*) Si $i \neq j$, on considère le P. H.: $\left(L^{\prime}=\mathbf{C}^{*} \times \prod_{l=2}^{r} G L\left(p_{l}\right) \times S O(q)\right.$, $\rho_{\left(1, p_{2}\right)} \oplus \rho_{\left(1, p_{2}\right)} \oplus \bigoplus_{l=2}^{r-1} \rho_{\left(p_{l}, p_{l+1}\right)} \oplus \tau_{\left(p_{r}, q\right)}, V=M\left(1, p_{2}\right) \oplus M\left(1, p_{2}\right) \oplus$ $\left.\bigoplus_{l=2}^{r-1} M\left(p_{l}, p_{l+1}\right) \oplus M\left(p_{r}, q\right)\right)$ (qui n'est pas de type parabolique, voir [Gy]), on vérifie facilement que le polynôme $\pi_{(i, j)}$ en est un invariant relatif. D'où l'existence du polynôme de Bernstein-Sato associé au polynôme $\pi_{(i, j)}$.

Notons que dans ce cas $b_{(i, j)}(s)=b_{(k, l)}(s)$ pour $1 \leq i<j \leq p_{1}$, $1 \leq k<l \leq p_{1}$.

Si $i=j$, on considère le P. H.:

$\left(L^{\prime}=\mathbf{C}^{*} \times \prod_{l=2}^{r} G L\left(p_{l}\right) \times S O(q), \rho_{\left(1, p_{2}\right)} \oplus \bigoplus_{l=2}^{r-1} \rho_{\left(p_{l}, p_{l+1}\right)} \oplus \tau_{\left(p_{r}, q\right)}, V=\right.$ $M\left(1, p_{2}\right) \oplus \bigoplus_{l=2}^{r-1} M\left(p_{l}, p_{l+1}\right) \oplus M\left(p_{r}, q\right)$ ) (qui est de type $B^{1}$ ou $D^{1}$ ), on vérifie facilement que le polynôme $\pi_{(i, i)}$ en est un invariant relatif. D'où l'existence du polynôme de Bernstein-Sato associé au polynôme $\pi_{(i, i)}$.

Notons que dans ce cas $b_{(i, i)}(s)=b_{(j, j)}(s)$ pour $1 \leq i, j \leq p_{1}$.

Et ainsi de suite.

Problème 5.3. Peut-on calculer $\mathcal{B}_{\theta}$ en fonction de $\mathcal{B}$ ?

Nous savons répondre à cette question dans le cas où $(L, \rho, V)$ est irréductible régulier (pas forcément commutatif) et dans le cas où $(L, \rho, V)$ est un P. H. Q-irréductible de type A.

On trouve $\mathcal{B}_{\theta}(s)=B(s) B\left(s+\varphi\left(p_{1}, \ldots, p_{r}, q\right)\right)$. 
Calcul Explicite 5.4.

Dans le cas $(G L(p) \times G L(q) \times S L(p), M(p, q) \oplus M(p, p)): \varphi(p, q)=q-p$.

Dans le cas $(G L(p) \times S O(q), M(p, q)): \varphi(p, q)=q-p$.

Dans le cas $(G L(2 p) \times S p(q), M(2 p, q)): \varphi(p, q)=q-2 p$.

Conjecture 5.5. Il existe des fonctions $\varphi_{k}\left(1 \leq k \leq N_{\theta}\right)$ sur $\left\{p_{1}, \ldots\right.$, $\left.p_{r}, q\right\}$ à valeurs dans $\mathbf{Q}$ telles que:

$$
\mathcal{B}_{\theta}(s)=\prod_{k=1}^{N_{\theta}} \mathcal{B}\left(s+\varphi_{k}\left(p_{1}, \ldots, p_{r}, q\right)\right)
$$

avec $\varphi_{1}\left(p_{1}, \ldots, p_{r}, q\right)=0$, i.e. $\mathcal{B}(s)$ divise $\mathcal{B}_{\theta}(s)$.

\section{$\S$ 6. Les polynômes harmoniques}

DÉfinition 6.1. Un polynôme $Q \in \mathbf{C}[V]$ est dit $M$-harmonique si:

1) $Q \in \mathbf{C}^{M}[V]=\mathbf{C}[\pi]$.

2) $\Delta_{0}^{\theta}(\partial) \cdot Q=0$.

L'ensemble $\mathcal{H}_{M}(V)$ désigne l'espace des polynômes $M$-harmoniques sur $V$.

Remarque 6.2.

i) Si le P. H. $(L, V)$ est commutatif, le groupe $M$ est trivial et dans ce cas la notion de polynômes $M$-harmonique coincide avec celle des polynômes harmoniques introduite dans $[R u-S c h]$.

ii) Un polynôme $P$ est $M$-harmonique si et seulement s'il existe un polynôme $Q \in \mathbf{C}[U]$ harmonique tel que $P(X)=Q(\pi(X))$.

iii) Soit le sous-groupe $H_{0}=\operatorname{Ker} \chi_{0}$. On a montré dans [Mor 2] que $\mathbf{C}^{H_{0}}[V]=\mathbf{C}\left[\Delta_{0}^{\theta}\right]$ et que l'idéal $\mathcal{I}_{M}$ engendré par $\Delta_{0}^{\theta}$ est l'ensemble des polynômes qui s'annullent sur l'ensemble $\Sigma_{\theta}=\left\{X \in V / \Delta_{0}^{\theta}(X)=0\right\}$.

iv) Les polynômes $M$-harmoniques sont les polynômes $Q \in \mathbf{C}[\pi]$ tels que $P(\partial) Q=0$ pour tout $P \in \mathcal{I}_{M}$.

v) Dans le cas du P. H. $\left(\mathbf{C}^{*} \times S O(q), \tau_{(1, q)}, M(1, q)\right)$ de type parabolique commutatif de type $B^{1}$, on retrouve la définition classique des polynômes harmoniques.

LEMME 6.3.

i) L'opérateur $\Delta_{0}^{\theta}(\partial)$ induit un isomorphisme de $H_{0}$-modules de $V_{a_{0}, \ldots, a_{d}}$ sur $V_{a_{0}-1, \ldots, a_{d}}$. 
ii) L'opérateur $\Delta_{0}^{\theta}(\partial)$ est nul sur $V_{0, a_{1}, \ldots, a_{d}}$.

iii) $\mathcal{H}_{M}(V)=\bigoplus_{\left(a_{1}, \ldots, a_{d}\right) \in \mathbf{N}^{d}} V_{0, a_{1}, \ldots, a_{d}}$.

iv) $\mathcal{I}_{M}=\bigoplus_{\substack{\left(a_{0}, \ldots, a_{d}\right) \in \mathbf{N}^{d+1} \\ 0<a_{0}}} V_{a_{0}, \ldots, a_{d}}$.

v) $\mathbf{C}^{M}[V]=\mathbf{C}[\pi]=\mathcal{I}_{M}(V) \otimes \mathcal{H}_{M}(V)$.

Démonstration. Vu que la représentation $(L, \mathbf{C}[\pi])$ est équivalente à la représentation $(\Gamma, \mathbf{C}[U])$, alors le lemme est une simple conséquence des résultats précedents, du Lemme 4.2 et le Théorème 4.4 de [Ru-Sch].

Remarque 6.4. Tout polynôme dans $\mathbf{C}[\pi]$ s'écrit d'une manière unique sous la forme $\sum\left[\Delta_{0}^{\theta}\right]^{k} Q_{k}$ où $Q_{k}$ est $M$-harmonique.

DÉFInition 6.5. Un polynômes $P$ est dit $\pi$-pluriharmonique si $\pi_{i}(\partial) P$ $=0$ pour tout $1 \leq i \leq k$. On note $\mathcal{H}_{\pi}(V)$ l'espace des polynômes $\pi$ pluriharmoniques et $\mathcal{I}_{\pi}(V)$ l'idéal de $\mathbf{C}[V]$ engendré par les $\pi_{i}$; i.e. $\mathcal{I}_{\pi}(V)=$ $\left\{P \in \mathbf{C}[V] / P(x)=O ; \forall x \in \pi^{-1}(0)\right\}$.

\section{Remarque 6.6.}

i) Soit la $\pi$-fibre $\pi^{-1}(0)=\left\{x \in V / \pi_{i}(x)=0 ; 1 \leq i \leq k\right\}$.

ii) $\mathrm{Vu}$ la nature des polynômes $\pi_{i}$, par le théorème des zéros de Hilbert, L'idéal $\mathcal{I}_{\pi}(V)$ de $\mathbf{C}[V]$ engendré par les $\pi_{i}$ est l'ensemble des polynômes qui s'annulent sur la $\pi$-fibre $\pi^{-1}(0)$.

\section{Proposition 6.7. $\quad \mathbf{C}[V]=\mathcal{I}_{\pi}(V) \oplus \mathcal{H}_{\pi}(V)$.}

Démonstration. Sur $\mathbf{C}[V]$, on a la forme bilinéaire symétrique non dégénérée $(P, Q)=(P(\partial) Q)(0)$. Il est clair que $\mathcal{H}_{\pi}(V)$ est le complémentaire orthogonal de $\mathcal{I}_{\pi}(V)$. Donc, $\mathbf{C}[V]=\mathcal{I}_{\pi}(V) \oplus^{\perp} \mathcal{H}_{\pi}(V)$.

\section{Proposition 6.8. $\quad \mathbf{C}[V]=\mathbf{C}[\pi] \cdot \mathcal{H}_{\pi}(V)$.}

Démonstration. Il suffit de montrer que tout polynôme homogène est dans $\mathbf{C}[\pi] \cdot \mathcal{H}_{\pi}(V)$. Soit $P$ un polynôme homogène de degré $m$. Par la proposition précédente, $P=P_{1}+P_{2}$ où $P_{1} \in \mathcal{I}_{\pi}(V)$ et $P_{2} \in \mathcal{H}_{\pi}(V)$. Donc, $P(x)=P_{1}(x)+\sum_{i=1}^{k} Q_{i}(x) \pi_{i}(x)$ avec $d^{0} Q_{i}=m-N$ et ainsi, on a le résultat par récurrence sur le degré de $P$. 
Proposition 6.9. $\quad \mathbf{C}[V]=\mathbf{C}[\pi] \otimes \mathcal{H}_{\pi}(V)$.

Démonstration. C'est une simple conséquence des propositions précédentes et du fait que les $\pi_{i}$ soient algébriquement indépendants.

Proposition 6.10. $\quad \mathbf{C}[V]=\mathcal{I}_{M}(V) \otimes \mathcal{H}_{M}(V) \otimes \mathcal{H}_{\pi}(V)$.

Démonstration. C'est une simple conséquence des propositions précédentes.

6.11. Les poids dominants des représentations irréductibles de $(L, \mathbf{C}[V])$ : Soit $(L, V)$ un P. H. de type parabolique classique régulier et soit $V=\bigoplus_{i=1}^{r} V_{i}$ la décomposition de $V$ en composantes irréductibles.

DÉfinition 6.11.1. On dit qu'un sous-ensemble $I$ de $A_{r}=\{1, \ldots, r\}$ est connexe si $i \in I$ et $i+1 \notin I \Rightarrow \sup I=i$.

On note $\mathcal{C}$ l'ensemble des parties connexes de $A_{r}=\{1, \ldots, r\}$.

Remarque 6.11.2. $\quad$ ard $\mathcal{C}=\frac{r(r+1)}{2}$.

Remarque 6.11.3. Pour tout $I \in \mathcal{C}$, l'action $\left(L, V_{I}=\bigoplus_{i \in I} V_{i}\right)$ est préhomogène de type parabolique classique de type $A$ si $r \notin I$ et de même type que $(L, V)$ si $r \in I$.

Notation 6.11.4. On note $\pi_{I}$ l'application de $V_{I}$ sur $U_{I}$ telle que $\left(L, U_{I}\right)$ soit le commutatif de $\left(L, V_{I}\right)$ et on note $\left\{\Delta_{0}^{I}, \ldots, \Delta_{d_{I}}^{I}\right\}$ le système fondamental des invariants relatifs de l'action du $I$-Borel $B_{I}$ de $L$ sur $V_{I}$.

Proposition 6.11.5. Les vecteurs dominants $P$ des représentations irréductibles qui apparaissent dans la décomposition de la représentation $(L, \mathbf{C}[V])$ sont:

$$
P=\prod_{I \in \mathcal{C}} \prod_{j=0}^{d_{I}}\left(\Delta_{j}^{I}\right)^{a_{j}^{I}}
$$

avec $a_{j}^{I} \in \mathbf{N}$ tel que si $P$ est homogène de dgré $d$,

$$
\sum_{I \in \mathcal{C}} \sum_{j=0}^{d_{I}}\left(d_{I}+1-j\right) a_{j}^{I}=d
$$


Proposition 6.11.6. Les vecteurs dominants $P$ des représentations irréductibles qui apparaissent dans la décomposition de la représentation $\left(L, \mathcal{H}_{\pi}(V)\right)$ sont:

$$
P=\prod_{I \in \mathcal{C}-\left\{A_{r}\right\}} \prod_{j=0}^{d_{I}}\left(\Delta_{j}^{I}\right)^{a_{j}^{I}}
$$

avec $a_{j}^{I} \in \mathbf{N}$ tel que si $P$ est homogène de dgré $d$,

$$
\sum_{I \in \mathcal{C}} \sum_{j=0}^{d_{I}}\left(d_{I}+1-j\right) a_{j}^{I}=d
$$

Démonstration. Il est clair que $P=\prod_{I \in \mathcal{C}-\left\{A_{r}\right\}} \prod_{j=0}^{d_{I}}\left(\Delta_{j}^{I}\right)^{a_{j}^{I}}$ est $\pi$ pluriharmonique.

Inversement, un vecteur dominant $P$ d'une représentation irréductible de $L$ sur $\left.\mathcal{H}_{\pi}(V)\right)$ est un invariant relatif de l'action d'un sous-groupe de Borel $B$ de $L$ sur $V$, donc, il est de la forme

$$
P=\prod_{I \in \mathcal{C}} \prod_{j=0}^{d_{I}}\left(\Delta_{j}^{I}\right)^{a_{j}^{I}}
$$

Comme $\left.P \in \mathcal{H}_{\pi}(V)\right), I \neq A_{r}$.

\section{$\S 7$. Le Cas des P. H. 1-Elementaires}

7.1 Rappellons la notion de P. H. 1-élémentaire introduite dans [Mor] et notons que cette notion généralise celle des $\mathrm{P}$. H. des chaines déscendantes introduite par F. Sato dans [Sa III].

Définition 7.1.1. On dit qu'un P. H. $(L, V)$ est 1-élémentaire de longueur $m+1$, s'il existe une décomposition $V=\bigoplus_{k=0}^{m} V_{k}$ et un point générique $J=\sum_{k=0}^{m} J_{k}$ tel qu'en posant $L=L_{0}$ et $L_{k}=\left(L_{k-1}\right)_{J_{k-1}}$ on ait:

1) $\left(L_{k}, V_{k}\right)$ quasi-irréductible régulier $k \in\{0, \ldots, m\}$.

2) $\left(L_{k}, \bigoplus_{j=t}^{r} V_{j}\right)$ quasi-irréductible non régulier pour $k+1 \leq t \leq j \leq r \leq$ $m$.

Remarque 7.1.2.

i) Un P. H. 1-élémentaire est régulier et un P. H. quasi-irréductible régulier est 1-élémentaire de longueur 1. 
ii) Le P. H. $\left(L_{k}, \bigoplus_{j=k}^{m} V_{j}\right)$ est 1-élémentaire de longueur $(m-k+1)$.

iii) Le P. H. $\left(L, \bigoplus_{j=0}^{k} V_{j}\right)$ est 1-élémentaire de longueur $(k+1)$.

iv) Dans un P. H. 1-élémentaire de type parabolique de longueur $m+1$, il y a exactement $m+1$ invariants relatifs fondamentaux $P_{0}, \ldots, P_{m}$ tels que chaque $P_{k}$ dépend (et seulement) des variables $\left(x_{0}, \ldots, x_{k}\right) \in$ $\bigoplus_{j=0}^{k} V_{j}(\operatorname{voir}[$ Mor 1]).

On note $\chi_{0}, \ldots, \chi_{m}$ les caractères correspondants.

\section{Notations 7.1.3.}

i) On considère dans toute la suite $(L, V)$ un P. H. de type parabolique 1-élémentaire classique et soit $V=\bigoplus_{k=0}^{m} V_{k}$ la décomposition correspondante.

ii) On pose $V^{t}=\bigoplus_{i=0}^{t} V_{i}$. Il est clair que $\left(L, V^{t}\right)$ est un P. H. de type parabolique classique 1-élémentaire.

Notons que $V^{m}=V$.

ThÉorème 7.1.4. Pour tout $t \in\{0, \ldots, m\}:$

1) Il existe une représentation $\left(\rho^{t}, U^{t}\right)$ de dimension $k_{t}$ d'un sous-groupe $\Gamma^{t}$ de $L$ et des polynômes homogènes algébriquement indépendants, de degré $N_{t}, \pi_{1}^{t}(x), \ldots, \pi_{k_{t}}^{t}(x)$ dans $\mathbf{C}[V]$ tels que:

i) $\left(\Gamma^{t}, \rho^{t}, U^{t}\right)$ soit un $P$. H. de type parabolique classique commutatif régulier.

ii) l'application polynomiale $\pi^{t}: V^{t} \rightarrow U^{t}$ définie par $\pi^{t}(x)=\left(\pi_{1}^{t}(x)\right.$, $\left.\ldots, \pi_{k}^{t}(x)\right)$ soit surjective.

iii) L'application $\pi^{t}$ est L-équivariante, i.e.: $\pi^{t}(g \cdot x)=\rho(g) \pi^{t}(x)$; $\forall g \in L, \forall x \in V^{t}$.

2) Il existe un homomorphisme injectif d'algèbres $\left(\pi^{t}\right)^{*}: \mathbf{C}\left[U^{t}\right] \rightarrow \mathbf{C}\left[V^{t}\right]$.

Démonstration. Voir [Mor 2].

\subsection{L'algèbre $\mathrm{C}[\pi]$}

DÉfinition 7.2.1. Par le théorème précédent, on a les ploynômes homogènes:

$$
\pi_{1}^{0}, \ldots, \pi_{k_{0}}^{0}, \pi_{1}^{1}, \ldots, \pi_{k_{1}}^{1}, \ldots, \pi_{1}^{t}, \ldots, \pi_{k_{t}}^{t}, \ldots, \pi_{1}^{m}, \ldots, \pi_{k_{m}}^{m}
$$


On note $\mathbf{C}[\pi]$ la sous algèbre de polynômes engendré par les $\pi_{j}^{t}(0 \leq t \leq m$ et $\left.1 \leq j \leq k_{t}\right)$ de l'algèbre $\mathbf{C}[V]$.

\section{Remarque 7.2.2.}

i) Si on pose $U=\bigoplus_{t=0}^{m} U^{t}$, alors l'algèbre $\mathbf{C}[\pi]$ est isomorphe à l'algèbre $\mathbf{C}[U]$.

ii) Il existe un sous-groupe $M_{0}$ de $L$ tel que:

$$
L=\Gamma_{0} \times \cdots \times \Gamma_{m} \times M_{0}
$$

(notons que $M_{0}$ peut être réduit à l'élément neutre).

iii) Chaque P. H. $\left(\Gamma^{t}, U^{t}\right)$ admet un et un seul invariant relatif fondamental, on le note $\Delta_{0}^{t}$.

iv) L'algèbre des polynômes $M_{0}$-invariants sur $V$ est $\mathbf{C}^{M_{0}}[V]=\mathbf{C}\left[\pi_{0}\left(V_{0}\right) \oplus\right.$ $\left.\bigoplus_{t=1}^{m} V_{t}\right]$.

v) Comme $\mathbf{C}[\pi] \cong \mathbf{C}[U]$ alors, $(L, \mathbf{C}[\pi])$ est équivalente à $(\Gamma, \mathbf{C}[U])$.

vi) On a $\mathbf{C}[U]=\bigotimes_{t=0}^{m} \mathbf{C}\left[U^{t}\right]$

Comme $\left(\Gamma^{t}, \mathbf{C}\left[U^{t}\right]\right)$ se décompose avec multiplicité un, il est clair que $\left(\sigma^{t}, W^{t}\right)$ intervient dans $\left(\Gamma^{t}, \mathbf{C}\left[U^{t}\right]\right)$ si et seulement si $\left(\bigotimes_{t=0}^{m} \sigma^{t}, \bigotimes_{t=0}^{m} \mathbf{C}\left[U^{t}\right]\right)$ intervient dans $(G, \mathbf{C}[U])$. On a alors:

$$
\mathbf{C}^{M_{0}}[V]=\mathbf{C}[\pi]=\bigoplus_{s \in \mathbf{N}}\left[\bigotimes_{t=0}^{m} \underset{\substack{\left(a_{0}^{t}, \ldots, a_{d_{t}}^{t}\right) \in \mathbf{N}^{d_{t}+1} \\ \sum_{t=0}^{m} a_{0}^{t}\left(d_{t}+1\right)+\cdots+a_{d_{t}}^{t}=s}}{\bigoplus_{a_{0}^{t}, \ldots, a_{d_{t}}^{t}}^{t}}\right]
$$

7.3. Les invariants relatifs fondamentaux du P. H. $(L, V)$ et fonction $\mathcal{B}$ Les invariants relatifs fondamentaux des P. H. $(L, \rho, V)$ sont $\left\{\Delta_{0}^{t}\left(\pi^{t}(X), 0 \leq t \leq m\right\}\right.$ où $\Delta_{0}^{t}$ est le seul invariant relatif fondamental du P. H. $\left(\Gamma^{t}, U^{t}\right)$. On a, alors, $P_{t}(x)=\Delta_{0}^{t}\left(\pi^{t}(x)\right.$.

On pose $P(x)=\prod_{t=0}^{m} P_{t}(x)$.

Notation 7.3.1. Pour $s=\left(s_{0}, \ldots, s_{m}\right) \in \mathbf{C}^{m+1}$, on pose:

i) $P_{0}^{s}(X)=\prod_{t=0}^{m} P_{t}(x)^{s_{t}}$.

ii) $s_{t}-1=\left(s_{0}, \ldots, s_{t-1}, s_{t}-1, s_{t+1}, \ldots, s_{m}\right)$ et $s(t)=\left(s_{0}, \ldots, s_{t}, s_{t+1}-\right.$ $\left.1, \ldots, s_{m}-1\right)$. 
THÉORÈME 7.3.2.

i) Il existe un polynôme $\mathcal{B}_{t}(s)$ de degré $N_{t} \cdot\left(d_{t}+1\right)$ tel que:

$$
P^{t}(\partial) \cdot P^{s}(x)=\mathcal{B}_{t}(s) \cdot P^{s_{t}-1}(x)
$$

$\left(d_{t}+1\right.$ est le degré de $\left.\Delta_{0}^{t}\right)$.

ii) Il existe un polynôme $B(s)$ de degré $\sum_{t=0}^{m} N_{t} \cdot\left(d_{t}+1\right)$ tel que:

$$
P(\partial) \cdot P(x)^{s}=\mathcal{B}(s) \cdot P(x)^{s-1}
$$

$\mathcal{B}(s)=\prod_{t=0}^{m} \mathcal{B}_{t}(s(t))$.

Démonstration.

i) Il est clair que pour tout $g \in L, g \cdot\left(P^{t}(\partial) \cdot P^{s}(x)\right)=\left(\prod_{j=0}^{m} \chi_{j}^{s_{t}-1}\right)\left(P^{t}(\partial)\right.$. $\left.P^{s}(x)\right)$ où $\chi_{j}$ désigne le caractère associé à l'invariant relatif $P_{j}$. Et ainsi, $P^{t}(\partial) \cdot P^{s}(x)$ est un invariant relatif de caractère $\prod_{j=0}^{m} \chi_{j}^{s_{t}-1}$, donc il ne différe que par une constante multiplicative de l'invariant relatif $\prod_{j=0}^{m} P_{j}^{s_{t}-1}$. On a alors:

$$
P^{t}(\partial) \cdot P^{s}(x)=\mathcal{B}_{t}(s) \prod_{j=0}^{m} P_{j}^{s_{t}-1}
$$

Il est clair que $\mathcal{B}_{t}(s)$ est un polynôme en $s$ de degré au plus $N_{t} \cdot\left(d_{t}+1\right)$. On sait, voir $[\mathrm{S}-\mathrm{K}]$, qu'il existe un polynôme $\mathcal{B}_{t}\left(s_{t}\right)$ de degré $N_{t} \cdot\left(d_{t}+1\right)$ tel que $P^{t}(\partial) \cdot P_{t}^{s_{t}}(x)=\mathcal{B}_{t}\left(s_{t}\right) P_{t}^{s_{t}-1}$.

Il est clair que $\mathcal{B}_{t}(s)=b_{t}\left(s_{t}\right)+\psi(s)$ d'où degré $\mathcal{B}_{t}(s)=N_{t} \cdot\left(d_{t}+1\right)$.

ii) C'est une simple conséquence de i).

7.4. Les polynômes harmoniques On note $\mathcal{I}(V)$ l'idéal engendré par les polynômes $P_{0}(x), \ldots, P_{m}(x)$. L'idéal $\mathcal{I}(V)$ est l'ensemble des polynômes s'annulant sur le lieu singulier $V-\Omega=S=\left\{x \in V / \prod_{t=0}^{m} P_{t}(x)=0\right\}$. On sait que $\mathbf{C}^{H}[V]=\mathbf{C}\left[P_{0}, \ldots, P_{m}\right]$ où $H=\bigcap_{t=0}^{m} \operatorname{Ker}\left(\chi_{t}\right)$; voir [Mor 2].

Démonstration. Comme $\prod_{t=0}^{m} P_{t}(x)$ est produit de polynômes irréductibles, alors $\sqrt{\mathcal{I}(V)}=\mathcal{I}(V)$ (le théorème des zéros de Hilbert).

DÉFINITION 7.4.1.

i) Un polynôme $Q \in \mathbf{C}[V]$ est dit harmonique si:

$P_{t}(\partial) \cdot Q=0 ; \forall 0 \leq t \leq m$

$\mathfrak{h}(V)$ désigne l'espace des polynômes harmoniques sur $V$. 
ii) Un polynôme $Q \in \mathbf{C}[V]$ est dit quasi-harmonique (en abrégé $Q$-harmonique si: $P \in \mathfrak{h}(V) \cap \mathbf{C}[\pi]$.

$\mathfrak{h}_{M_{0}}(V)$ désigne l'espace des polynômes $Q$-harmoniques sur $V$.

iii) Un polynôme $Q \in \mathbf{C}[V]$ est dit pluriharmonique si: $\pi_{i}^{t}(\partial) \cdot Q=0$; $\forall 0 \leq t \leq m$ et $1 \leq i \leq k_{t}$ i.e. $P$ est $\pi^{t}$-pluriharmonique pour $0 \leq t \leq$ $m)$.

$\mathfrak{h}_{\pi}(V)$ désigne l'espace des polynômes pluriharmoniques sur $V$.

Remarque 7.4.2.

i) Si le P. H. $(L, \rho, V)$ est Q-irréductible, un polynôme harmonique est $Q$ harmonique et un polynome pluriharmonique est $\pi$-pluriharmonique.

ii) Sur un P. H. commutatif les deux premières notions sont confondues et $\mathfrak{h}_{\pi}(V)=\mathbf{C}$.

iii) on a $\mathfrak{h}_{\pi}(V) \subset \mathfrak{h}(V)$.

Notation 7.4.3. Comme $d_{m} \geq d_{m-1} \geq \ldots \geq d_{0}$, on note:

$$
\begin{aligned}
\mathcal{N} & =\left\{A=\left(a_{j}^{i}\right)_{\substack{0 \leq i \leq m \\
0 \leq j \leq d m}} \in M\left(m+1, d_{m}+1 ; \mathbf{N}\right) / a_{j}^{i}=0 \text { pour } j>d_{i}\right\} \\
\mathcal{N}^{*} & =\left\{\left(a_{j}^{i}\right)_{\substack{0 \leq i \leq m \\
0 \leq j \leq d_{m}}} \in \mathcal{N} / a_{0}^{i}=0 \text { pour } 0 \leq i \leq m\right\} \\
\text { et } \quad \mathcal{N}^{+} & =\left\{\left(a_{j}^{i}\right)_{\substack{0 \leq i \leq m \\
0 \leq j \leq d m}} \text { in } \mathcal{N} / a_{0}^{i}>0 \text { pour } 0 \leq i \leq m\right\}
\end{aligned}
$$

Si $A \in \mathcal{N}$, on note $A-1=\left(b_{j}^{i}\right)_{\substack{0 \leq i \leq m \\ 0 \leq j \leq d_{m}}}$ avec $b_{0}^{i}=a_{0}^{i}-1($ pour $0 \leq i \leq m)$ et $b_{j}^{i}=a_{j}^{i}$ pour $\left(1 \leq i \leq m\right.$ et $\left.1 \leq j \leq d_{m}\right)$

Remarque 7.4.4. Il est clair qu'une représentation irréductible $\sigma$ intervenant dans la décomposition de $(L, \mathbf{C}[\pi])$ est paramétrée par une matrice $A \in \mathcal{N}$. On note $U_{A}$ l'espace d'une telle représentation.

\section{LEMME 7.4.5.}

i) L'opérateur $P(\partial)$ induit un isomorphisme de $H$-modules de $U_{A}$ sur $U_{A-1}$.

ii) Pour tout $A \in \mathcal{N}^{*}$, l'opérateur $P(\partial)$ est nul sur $U_{A}$.

iii) $\mathfrak{h}_{M_{0}}(V)=\bigoplus_{A \in \mathcal{N}^{*}} U_{A}$.

iv) $\mathcal{I}(V)=\bigoplus_{A \in \mathcal{N}^{+}} U_{A}$.

v) $\mathbf{C}[\pi]=\mathcal{I}(V) \oplus \mathfrak{h}_{M_{0}}(V)$. 
Démonstration. Vu que la représentation $(L, \mathbf{C}[\pi])$ est équivalente à la représentation $(L, \mathbf{C}[U])$, alors le lemme est une simple conséquence des résultats précedents.

Notons $\mathcal{J}_{\pi}(V)$ l'idéal de $\mathbf{C}[V]$ engendré par les $\pi_{i}^{t}(0 \leq t \leq m$ et $\left.0 \leq i \leq k_{t}\right)$.

\section{LEMME 7.4.6. $\quad \mathbf{C}[V]=\mathcal{J}_{\pi}(V) \oplus \mathcal{H}_{\pi}(V)$.}

Démonstration. L'espace $\mathcal{H}_{\pi}(V)$ est le complémentaire orthogonal de $\mathcal{J}_{\pi}(V)$

\section{Proposition 7.4.7. $\quad \mathbf{C}[V]=\mathbf{C}[\pi] \cdot \mathcal{H}_{\pi}(V)$.}

Démonstration. Il suffit de montrer que tout polynôme homogène est dans $\mathbf{C}[\pi] \cdot \mathcal{H}_{\pi}(V)$.

Soit $P$ un polynôme homogène de degré $d$. Par la proposition précédente, $P=P_{1}+P_{2}$ où $P_{1} \in \mathcal{J}_{\pi}(V)$ et $P_{2} \in \mathcal{H}_{\pi}(V)$.

Donc, $P(x)=P_{1}(x)+\sum_{0 \leq t \leq m} \sum_{i=1}^{k_{t}} Q_{i}^{t}(x) \pi_{i}^{t}(x)$ avec $d^{0} Q_{i}^{t}=d-N_{t}$ et ainsi, on a le résultat par récurrence sur le degré de $P$.

Proposition 7.4.8. $\quad \mathbf{C}[V]=\mathbf{C}[\pi] \otimes \mathcal{H}_{\pi}(V)$.

Démonstration. C'est une simple conséquence des propositions précédentes et du fait que les $\pi_{i}^{t}$ soient algébriquement indépendants.

Proposition 7.4.9. $\quad \mathbf{C}[V]=\mathcal{I}(V) \otimes \mathcal{H}_{M_{0}}(V) \otimes \mathcal{H}_{\pi}(V)$.

Démonstration. C'est une simple conséquence des propositions précédentes.

Conclusion 7.4.10. $\mathfrak{h}(V)=\left(\bigoplus_{A \in \mathcal{N}^{*}} U_{A}\right) \otimes \mathcal{H}_{\pi}(V)$.

Ainsi, $\mathbf{C}[V]=\mathcal{I}(V) \otimes \mathfrak{h}(V)$.

\section{BIBLIOGRAPHIE}

[Bo] N. Bourbaki, Algèbre de Lie, chap. 7-8, Hermann.

[Ko-Sah] B. Kostant and S. Sahi, The Capelli identity, tube domains and the generalized laplace transform, Adv. in Math., 87 (1991), 71-92.

[Ho] R. Howe, Perspectives in invariant theory, I. M. C. P., 8 (1995), 1-182.

[Ka] V. G. Kac, Some remarks on nilpotent orbits, J. Algebras, 64 (1980). 
[Ho-Um] R. How and T. Umeda, The Capelli identity, the double theorem, and multiplicity-free actions, Math. Ann., 290 (1991).

[K-V] M. Kashiwara and M. Vergne, On the Segal-Shale-Weil representation and harmonic polynomials, Inv. Math., 44 (1978), 1-47.

[Lu] D. Luna, Slices etales, Bull. Cos. Math. France Mémoire, 33 (1973), 81-105.

[Mor 1] A. L. Mortajine, Classification des espaces préhomogènes de type parabolique réguliers et de leurs invariants relatifs, Travaux en cours, 40 (1991).

[Mor 2] A. L. Mortajine, Z-graduation de type parabolique et théorie des invariants, Prépublication de l'IECN 1/97 (1997).

[Mu-Ru-Sch] I. Muller, H. Rubenthaler and G. Schiffmann, Structure des espaces préhomogènes associés à certaines algèbres de Lie graduées, Math. Ann., 274 (1986).

[Mum] D. Mumford, Geometric invariant theory, Academic Press, New York, 1965.

[Po] V. Popov, Groups, generators, syzygies, and orbits in invariant theory, Transl. Mono., 100, American Math. Coc., Providence, R. I., 145-156.

$[\mathrm{Ru}] \quad \mathrm{H}$. Rubenthaler, Espaces préhomogènes de type parabolique, thèse d'Etat,, IRMA (1982).

[Ru-Sch] H. Rubenthaler and G. Schiffmann, Opérateurs différentiels de Shimura et espaces préhomogènes, Inv. Math., 90 (1987), 409-488.

[Sa III] F. Sato, Zeta function associated with puch mogenaous vector spaces III: Einsenstein series of indefinite quadratic forms, Ann. Math., 116 (1982).

[Sa I] F. Sato, Zeta function of several variables associated with prehomogeneous vector spaces I: functional equation, Tohoko Math. Japan, 34 (1982), 437-483.

[FS] F. Sato, Zeta function with polynomial coefficients associated with prehomogeneous vector spaces, Preprint.

[S-K] Sato and T. Kimura, Classification of irreducible prehomogeneous vector spaces and their relative invariants, Nagoya Math. J., 65 (1877).

[Shim] G. Shimura, On differential operators attached to certain representations of classical groups, Inv. Math., 77 (1984), 463-442.

[Sp] Springer, Invariant theory, L. N. M., 585, 1977.

[Sr] F. Servidio, Prehomogeneous spaces and varieties, TAMS, 76 (1973), 421-444.

[V] E. B. Vinberg, On the classification of the nilpotent elements of graded Lie algebras, Soviet Math. Dokl., 16 (1975), 1517-1520.

[V-K] E. B. Vinberg and Kimelfeld, Homogeneous domains in flag manifolds and spherical subgroups of semi-simple Lie groups, Func. Anal. Appli., 12 (1978), 12-19.

[W] A. Weyl, The classical groups, Princeton University Press, Princeton, N. J., 1946. 
Département de Mathématiques-URA 750 CNRS

Université de Nancy

1, BP 239, 54506 Vandoeuvre les Nancy Cedex mortajin@iecn.u-nancy.fr 